\title{
An Adult Case of Primary Rhabdomyosarcoma of the Trachea with Resection of Seven Cartilage Rings and Primary Reconstruction
}

\author{
Ken Miwa ${ }^{1}$ Yuji Taniguchi ${ }^{1}$ Hiroshige Nakamura ${ }^{1}$ \\ ${ }^{1}$ Department of General Thoracic Surgery, Tottori University Hospital, \\ Yonago, Japan \\ Address for correspondence Ken Miwa, Department of General \\ Thoracic Surgery, Tottori University Hospital, 36-1 Nishi-Cho, Yonago \\ 683-8504, Japan (e-mail: miwa@med.tottori-u.ac.jp). \\ Thorac Cardiovasc Surg 2012;60(S2):e19-e21.
}

\begin{abstract}
Keywords

- tracheal tumor

- rhabdomyosarcoma

- trachea

A 74-year-old female consulted a local clinic with bloody sputum and dyspnea. Bronchoscopy revealed a tumor with $\sim 80 \%$ stenosis in the trachea. She was referred to our hospital. Biopsy findings suggested primary rhabdomyosarcoma of the trachea. Cervical computed tomography (CT) revealed a tumor involving the 3rd to 9th tracheal cartilages. Tubular resection of the 3rd to 9th tracheal cartilages and tracheal reconstruction were performed. Finally, a pathological diagnosis of rhabdomyosarcoma was made. Primary rhabdomyosarcoma of the trachea is extremely rare.
\end{abstract}

\section{Introduction}

The incidence of adult rhabdomyosarcoma is 2 to $5 \%$ in all sarcoma patients. ${ }^{1,2}$ There is only one case report of resection/reconstruction for primary adult rhabdomyosarcoma of the trachea. ${ }^{3}$ Rhabdomyosarcoma is highly malignant, showing dissemination in the early stage. ${ }^{4}$ The 5 -year survival rate in patients receiving multidisciplinary treatment ranges from 31 to $44 \% ; 2,4$ the prognosis is not favorable. In this study, we report a patient with primary adult rhabdomyosarcoma of the trachea in whom resection/reconstruction of seven tracheal cartilage rings were performed, and review the literature.

\section{Case}

A 74-year-old female. Stridor had sometimes occurred since 2008. In 2010, exertional dyspnea was noted. She consulted a local clinic, and drug therapy for asthma was administered. In February 2011, she consulted another hospital with bloody sputum and the exacerbation of dyspnea. Bronchoscopy revealed a tracheal tumor. She was referred to another hospital. To treat the tracheal tumor, she was admitted to the Department of Respiratory Medicine in our hospital. Bronchoscopy showed an extensive, hemorrhagic tumor in the membranous region of the upper trachea. The percent stenosis was $\sim 80 \%$. Biopsy findings suggested rhabdomyosarcoma. Cervical computed tomography (CT) revealed a protruding tracheal tumor involving the 3rd to 9th tracheal cartilages. The border with the esophagus was unclear (-Fig. 1). Progressive dyspnea was present, and there was no metastasis; therefore, emergency surgery was selected. For surgery, percutaneous cardiopulmonary support (PCPS) was on stand by, and anesthesia was induced using a laryngeal mask. Initially, a collar-like cervical incision was performed. The frozen-section examination of a lymph node in the left trachea suggested metastasis from rhabdomyosarcoma. On palpation, the esophageal infiltration of the tumor was unclear. However, as the trachea could be secured on the carinal side of the tumor, simultaneous resection was considered to be possible, and median sternotomy was additionally performed. The trachea was resected at the superior margin of the 10th tracheal cartilage, which was macroscopically the carinal-side margin of the tumor. Intubation from operative field was conducted, and respiratory control was started. There was no esophageal tumor infiltration. Macroscopically, further resection of the trachea was impossible and unclear whether reconstruction could be performed safely. So, we did not examine microscopic margins on rapid pathological diagnosis. On the oral side, tubular resection of received

November 25, 2011

accepted after revision

January 17, 2012

published online

November 8, 2012 (c) 2012 Georg Thieme Verlag KG Stuttgart · New York
DOI http://dx.doi.org/ 10.1055/s-0032-1311541. ISSN 2194-7635. 

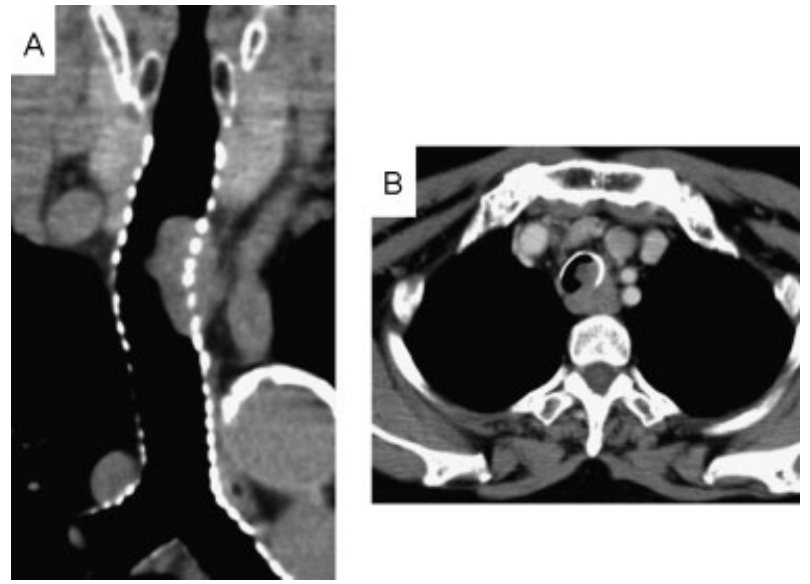

Figure 1 A chest CT shows locally advanced tracheal tumor extensively invading to the extratracheal tissues. (A) coronal imaging of trachea, (B) transaxial imaging.

seven cartilage rings with macroscopic margins was performed at the superior margin of the 3rd tracheal cartilage to complete tumorectomy. To relieve tension at the anastomotic site, hilar exfoliation and pulmonary ligament cut-off were performed so that the trachea was sustained. For tracheal reconstruction, end-to-end anastomosis was conducted employing single-node suture with 3-OPDS (12 stitches). The anastomotic site was covered with thymic fat. A tube from operative field was removed prior to tracheal anastomosis, and a tube for oral intubation was induced. The resected trachea measured $35 \mathrm{~mm}$ in length. The tumor, measuring $34 \times 32 \times 16 \mathrm{~mm}$, involved an extra-trachealwall area (-Fig. 2). Histopathologically, bundle to alveolar tumor cells with spindle nuclei and cytoplasm were present below the tracheal mucosa. Immunostaining showed positive reactions to vimentin and desmin, as well as negative reactions to cytokeratin AE1/AE3, $\alpha \mathrm{SMA}$, myoglobin, myogenin, CD99, NSE, and EMA, suggesting primary rhabdomyosarcoma of the trachea (alveolar type) (- Fig. 3 ). The oral- and carinalside margins of the resected tracheal specimen were positive for tumor cells. To relieve tension at the anastomotic tracheal site, the cervix was fixed with a brace for 3 weeks after

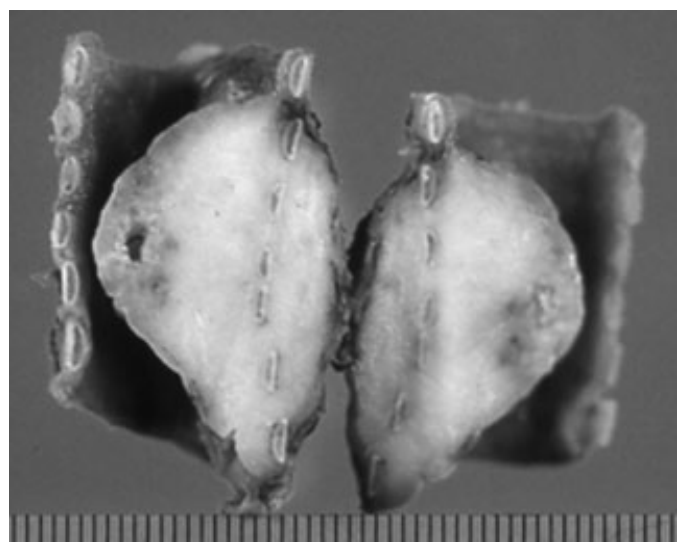

Figure 2 The resected tumor shows the extensive involvement of six cartilages. The length of the resected trachea was $35 \mathrm{~mm}$.

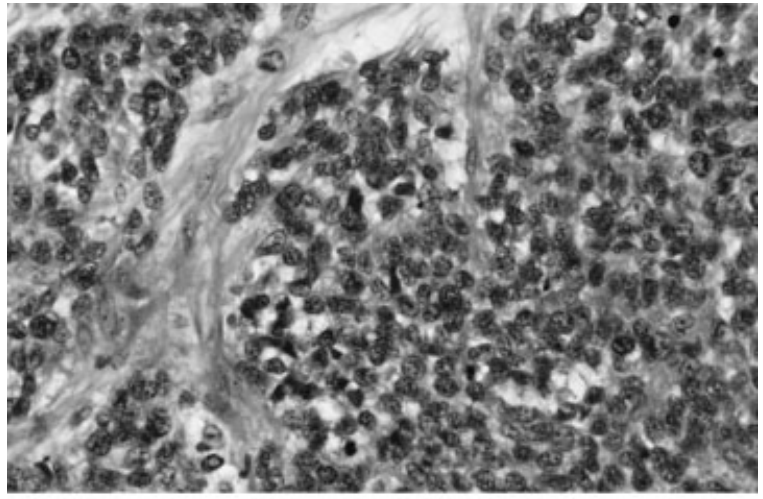

H.E $\times 100$

Figure 3 Rhabdomyosarcoma showing spindle-shaped cells invading along just beneath the tracheal mucosa.

surgery. The patient was discharged 28 days after the surgery. Postoperative adjuvant therapy was not conducted. Bronchoscopy did not reveal any stenosis at the anastomotic site, and healing was achieved. We must follow closely future.

\section{Discussion}

Rhabdomyosarcoma is frequently detected as a malignant tumor in children. However, the incidence of adult rhabdomyosarcoma ranges from 2 to $5 \%$ in all sarcoma patients. ${ }^{1,2}$ To our knowledge, only Ho et $\mathrm{al}^{5}$ published a case report in which resection/reconstruction for primary adult rhabdomyosarcoma of the trachea were performed, as conducted in the present case. Rhabdomyosarcoma is highly malignant, showing dissemination in the early stage. ${ }^{4}$ In particular, the present patient had alveolar-type rhabdomyosarcoma, of which the prognosis is unfavorable. ${ }^{4}$ Regional lymph node metastasis was detected 3 years after the symptom appearance, although tumor progression was slightly slow. Resection of the trachea with macroscopic margins was performed. As a result, the margins of the resected specimen were positive for tumor cells. However, if additional resection of one tracheal cartilage ring is conducted on both the oral and carinal sides, this procedure may deviate from resection of eight tracheal cartilage rings, which is the limitation of safe end-to-end anastomosis; ${ }^{5}$ it is unclear whether reconstruction can be performed safely. In addition, the tumor involved an extensive area below the tracheal mucosa; we cannot assure that the margins of the resected specimen will be negative for tumor cells. According to a study, ${ }^{6}$ when surgeons consider further resection of the trachea impossible, positive findings on rapid pathological diagnosis may be acceptable. In addition, Gaissert et $\mathrm{al}^{7}$ also indicated that positive reactions at the margins of the resected specimen on pathological diagnosis should be accepted when macroscopic margins are present. In 26 of 77 patients who had undergone resection of the trachea, the margins of the resected specimen were positive for tumor cells. In the present case, lymph node metastasis was detected on frozen-section examination. Based on this, the extent of tracheal resection was established at the macroscopic margins. 
Concerning treatment, there are several chemotherapeutic protocols in the field of pediatrics. The survival rate is $70 \%$ or more. On the other hand, treatment guidelines for adults consist of following multidisciplinary treatment: ${ }^{8}$ extended resection, radiotherapy for residual lesions, and combination chemotherapy. The response rate is low, ${ }^{5}$ and the 5 -year survival rate ranges from 31 to $44 \%^{2,4}$ Considering our patient's age (over 70 years) and the influence of radiotherapy on the tracheal reconstruction site, adjuvant therapy was not conducted. During the 3-month postoperative follow-up, there has been no relapse. However, periodic CT and bronchoscopy should be performed in the future.

\section{References}

1 Hawkins WG, Hoos A, Antonescu CR, et al. Clinicopathologic analysis of patients with adult rhabdomyosarcoma. Cancer 2001;91(4):794-803
2 Ferrari A, Dileo P, Casanova M, et al. Rhabdomyosarcoma in adults. A retrospective analysis of 171 patients treated at a single institution. Cancer 2003;98(3):571-580

3 Ho KL, Rassekh ZS. Rhabdomyosarcoma of the trachea: first reported case. Hum Pathol 1980;11(5, Suppl):572-574

4 Esnaola NF, Rubin BP, Baldini EH, et al. Response to chemotherapy and predictors of survival in adult rhabdomyosarcoma. Ann Surg 2001;234(2):215-223

5 Mulliken JB, Grillo HC. The limits of tracheal resection with primary anastomosis: further anatomical studies in man. J Thorac Cardiovasc Surg 1968;55(3):418-421

6 Liu XY, Liu FY, Wang Z, Chen G. Management and surgical resection for tumors of the trachea and carina: experience with 32 patients. World J Surg 2009;33(12):2593-2598

7 Gaissert HA, Grillo HC, Shadmehr MB, et al. Uncommon primary tracheal tumors. Ann Thorac Surg 2006;82(1):268-272, discussion 272-273

8 Crist W, Gehan EA, Ragab AH, et al. The third intergroup rhabdomyosarcoma study. J Clin Oncol 1995;13(3):610-630 